\title{
Repercussões de doença renal crônica na rotina familiar de crianças em hemodiálise
}

\section{Repercussions of chronic kidney disease in the family routine of children on hemodialysis}

\author{
Heloisa Sâmella Santos dos Santos ${ }^{1}$, Larice Pinto de Almeida ${ }^{2}$, \\ Fernanda do Socorro Carvalho da Silva ${ }^{3}$, Elson Ferreira Costa ${ }^{4}$, \\ Luísa Sousa Monteiro Oliveira ${ }^{5}$
}

http://dx.doi.org/10.11606/issn.2238-6149.v31i1-3p24-30

\begin{abstract}
Santos HSS, Almeida LP, Silva FSC, Costa EF, Oliveira LSM. Repercussões de doença renal crônica na rotina familiar de crianças em hemodiálise. Rev Ter Ocup Univ São Paulo. 2020 jan.-dez.;31(1-3):24-30.
\end{abstract}

RESUMO: Nesta pesquisa objetivou-se analisar as repercussões da DRC na rotina familiar de crianças em tratamento de hemodiálise. Trata-se de um estudo exploratório, com abordagem qualitativa dos dados. Participaram 10 responsáveis/cuidadores principais de crianças com DRC em tratamento de hemodiálise, em um hospital de referência no tratamento de doenças renais pediátricas em Belém/PA. As categorias analisadas evidenciam a percepção das cuidadoras sobre as principais alterações na rotina familiar, são elas: "A percepção das cuidadoras sobre as principais alterações na rotina familiar"; "Os dias mais preocupantes" e "Os dias mais tranquilos". Seus conteúdos incluem o abandono de atividades significativas, alterações nas Atividades de Vida Diária como alimentação, a mudança de cidade e consequentemente o afastamento da criança e cuidadora dos demais membros da família. Considera-se relevante o conhecimento deste tema pelos terapeutas ocupacionais pois, uma vez conhecidas as repercussões ocupacionais da DRC na rotina familiar as intervenções podem ser aprimoradas e favorecer uma atenção mais humanizada e sistematizada.

DESCRITORES: Doença renal crônica; Crianças;Família.
Santos HSS, Almeida LP, Silva FSC, Costa EF, Oliveira LSM. Repercussions of chronic kidney disease in the family routine of children on hemodialysis. Rev Ter Ocup Univ São Paulo. 2020 Jan-Dec.;31(1-3):24-30.

\begin{abstract}
The objective of this research was to analyze the repercussions of Chronic Kidney Disease (CKD) on the routines of families with children on hemodialysis treatment. It is an exploratory study and the method used was the qualitative approach. The 10 participants were responsible for/the primary caretaker of children with CKD undergoing hemodialysis treatment in a reference hospital for the treatment of pediatric kidney diseases, located in Belém/PA. The categories which emerged during the research process pointed out the main changes that occurred in the family routine according to the perception of the caretaker. Such categories were divided into: "The perception of the caretakers on the main changes in the family routine", "The most worrying days", and "The calmest days". These categories include the abandonment of significant activities, modifications in the daily life routine such as eating habits, moving to a different city and hence the separation of the child and the caretaker from other family members. Thus, the knowledge of this rarely explored topic by occupational therapists is considered relevant as, once the occupational repercussions of CKD in the family routine are known, interventions can be enhanced, which could support a more humanized and systematic care.
\end{abstract}

KEYWORDS: Chronic kidney disease; Children; Family.

Artigo referente ao TCC de Bacharel em TO, apresentado à Universidade Federal do Pará (UFPA), Faculdade de Fisioterapia e Terapia Ocupacional do Instituto de Ciência da Saúde.

1. Terapeuta ocupacional pela Universidade Federal do Pará (UFPA). https://orcid.org/0000-0002-6085-6156. E-mail:heloisasantos@outlook.com.

2. Terapeuta ocupacional pela Universidade Federal do Pará (UFPA). https://orcid.org/0000-0002-6502-8560. E-mail: laricepinto@gmail.com.

3. Secretaria de Estado de Saúde Pública (SESPA), Fundação Santa Casa de Misericórdia do Pará (FSCMP). https://orcid.org/0000-0002-8460-1078. E-mail: fernandato23@hotmail.com.

4. Universidade do Estado do Pará (UEPA), Doutor em Teoria e Pesquisa do Comportamento pela Universidade Federal do Pará (UFPA). https://orcid.org/0000-0003-4115-9029. E-mail: elsonfcosta@gmail.com.

5. Mestra em Teoria e Pesquisa do Comportamento pela Universidade Federal do Pará (UFPA), Professora Assistente I da UFPA, vinculada ao Instituto de Ciências da Saúde, Faculdade de Fisioterapia e Terapia Ocupacional. https://orcid.org/0000-0002-3120-1839. E-mail: luisatomonteiro@gmail.com.

Endereço para correspondência: Luísa Sousa Monteiro Oliveira. Universidade Federal do Pará. Faculdade de Fisioterapia e Terapia Ocupacional, Instituto de Ciências da Saúde. Curso de Terapia Ocupacional. R. Augusto Corrêa, 1 - Guamá, Belém,PA. CEP 66075-110. 


\section{INTRODUÇÃO}

A s doenças crônicas são definidas como um grupo de problemas de saúde que persistem por longos períodos de tempo e requerem administração e cuidados permanentes, podendo ou não ser superados, assim como podem ou não deixar sequelas ${ }^{1}$. As implicações desse tipo de doença na infância são acompanhadas de particularidades, que abrangem dificuldades estruturais, instabilidade emocional e modificam intensamente o cotidiano da criança e de sua família. Isso pode resultar em agravantes como longos períodos de hospitalização, reinternações, tratamentos invasivos com efeitos colaterais adversos, afastamento da família, alterações na rotina, dificuldades financeiras, entre outros ${ }^{1,2}$.

A família é um grupo de indivíduos em associação íntima e contínua que interagem constantemente e, ainda, individual e coletivamente com a comunidade e grupos culturais dos quais fazem parte e pode ser influenciada por diversos fatores, neste estudo destaca-se os casos em que um membro da família é acometido por uma doença crônica ${ }^{3}$. Compreende-se que a família tem participação primordial no processo de tratamento e acompanhamento como ocorre nos casos de Doença Renal Crônica (DRC). Diante dessa realidade, a família experimenta desorganização intensa de sua rotina, além do sofrimento e do medo gerados pelos limites impostos à convivência ${ }^{1}$.

De acordo com a Estrutura da Prática da Terapia Ocupacional: domínio e processo, a rotina se configura como padrões de comportamento que são observáveis, regulares e repetitivos e que fornecem a estrutura para a vida diária. Ela pode ser satisfatória, promocional ou prejudicial ${ }^{4}$. Sendo assim, a rotina familiar é desenvolvida e estabelecida de acordo com os papéis, hábitos e funções designadas por cada membro familiar, no qual as ocupações são organizadas e desenvolvidas de forma harmoniosa.

Para a família da criança com DRC em tratamento de hemodiálise, a rotina familiar sofre grandes adaptações decorrentes das necessidades do tratamento, tais como; viagens intermunicipais semanais ou até mudança de cidade para facilitar o acesso ao tratamento, sendo importante aos profissionais o conhecimento de tais situações. Logo, esta pesquisa objetivou analisar as repercussões da doença renal crônica na rotina familiar de crianças em tratamento de hemodiálise.

\section{PROCEDIMENTOS METODOLÓGICOS}

Trata-se de um estudo exploratório, com abordagem qualitativa dos dados. Na metodologia qualitativa aplicada à saúde, no contexto da pesquisa, é empregada a concepção trazida das Ciências Humanas, que não busca entender o fenômeno em si, mas entender o seu significado individual ou coletivo para a vida das pessoas 5 .

A pesquisa foi realizada em um hospital de referência do município de Belém-PA. O setor de Terapia Renal Substitutiva (TRS). Este serviço, inaugurado em outubro de 2011, oferece atendimento a crianças e adolescentes menores de 18 anos com patologias renais agudas e crônicas. Apresenta capacidade de atendimento para 32 usuários e possui serviços de consulta com nefrologista pediátrico, hemodiálise pediátrica, ambulatórios, enfermarias e interconsultas ${ }^{6}$.

No intuito de conhecer as repercussões da DRC infantil na rotina familiar foi elaborada uma entrevista semiestruturada, contemplando: "Dados pessoais; Fatores socioeconômicos; Histórico clínico da criança e a Dinâmica da rotina familiar". A entrevista foi aplicada junto ao responsável da criança, e nos casos de desconhecimento do entrevistado sobre algum aspecto clínico foram necessárias consultas aos prontuários. Participaram da pesquisa 10 responsáveis/cuidadores principais, que leram e concordaram com o Termo de Consentimento Livre e Esclarecido (TCLE).

A análise das informações coletadas seguiu procedimentos qualitativos de análise do conteúdo de Bardin. Esta sugere uma organização para a aplicabilidade coerente do método que se divide em três fases, a saber: a pré-análise, a exploração do material e, por fim o tratamento dos resultados ou sua interpretação ${ }^{7}$.

O Quadro 1 mostra a caracterização dos participantes da pesquisa. As entrevistas foram realizadas nos meses de setembro e outubro de 2017, antecedidas pela apresentação da pesquisa para os profissionais de saúde e os responsáveis das crianças. As entrevistas ocorreram nos períodos matutino e vespertino em uma sala reservada, cedida pelo setor de TRS, geralmente tinham duração média de 30 minutos e ocorriam de forma concomitante aos procedimentos de hemodiálise da criança.

A pesquisa teve a supervisão das terapeutas ocupacionais do setor de TRS que facilitaram a comunicação com os responsáveis das crianças e favoreceram a relação de confiabilidade entre pesquisadoras e participantes, e a compreensão sobre a importância dos seus relatos.

\section{RESULTADOS}

A partir das informações coletadas foram identificadas as principais repercussões da DRC na rotina familiar de crianças em tratamento de hemodiálise, de acordo com a percepção do responsável/cuidador principal que acompanhava e participava ativamente do tratamento. 
Todos os participantes foram do sexo feminino, sendo 9 mães e 1 avó paterna, com idades entre 28 e 56 anos, no intuito de preservar suas identidades adotou-se a nomenclatura "C" com a numeração correspondente à ordem de realização da entrevista. A partir da análise do conteúdo das entrevistas emergiram três categorias, que são: "A percepção das cuidadoras sobre as principais alterações na rotina familiar"; "Os dias mais preocupantes" e "Os dias mais tranquilos".

\section{A percepção das cuidadoras sobre as principais alterações na rotina familiar}

A doença crônica na infância pode trazer impacto significativo ao funcionamento familiar (p. 244) ${ }^{8} \mathrm{e}$ isso foi confirmado nos conteúdo das entrevistas. Muitas atividades deixaram de fazer parte da rotina familiar, pois a criança realizava o procedimento de hemodiálise no mínimo 3 vezes por semana. Isso gerava a necessária reorganização na rotina de todos os membros da família, tanto do responsável/ cuidador principal como dos que ficavam em casa realizando as atividades necessárias do dia a dia.

A criança adoecida torna-se alvo da atenção dos familiares, modificando-se as hierarquias preexistentes à doença. Quando a criança é acometida por uma doença grave, os pais experimentam transformações que podem determinar a perda de atividades habitualmente realizadas por eles. Isso significa se abster de viagens, atividades sociais, estudar, ficar mais tempo com outros familiares (inclusive outros filhos) e até mesmo do trabalho (p. 70) ${ }^{9}$. Os relatos abaixo exemplificam tais mudanças:

"Passeávamos mais, saíamos para comer fora. Hoje ficamos mais em casa e tudo depende da rotina de tratamento". (C1)

"Nós fazíamos muitas atividades juntos em família. Meu esposo teve que largar o trabalho e eu tive que adaptar meus horários sem contar que nosso outro filho nos cobra mais atenção”. (C3)

Alguns autores relatam, que uma das primeiras mudanças ocorridas no ambiente familiar é o abandono do emprego por um dos pais, geralmente a mãe para acompanhar o filho durante a hospitalização, as consultas frequentes e na realização de exames (p. 783) ${ }^{10}$.

As cuidadoras foram indagadas como os demais integrantes da família veem o tratamento e como lidam com a DRC:

"É uma necessidade... (a hemodiálise). A família já se adaptou, mas cada um ainda tem as suas queixas". (C3)
"É uma lida necessária (a hemodiálise). A família entende e o irmão dele ajuda muito, pois ele ficava muito triste e ansioso. O pai dele que ainda chora às vezes". (C1)

Há situações em que os familiares não entendem as peculiaridades da doença ou até mesmo não convivem diretamente com a rotina do tratamento, devido à necessidade da pessoa com doença crônica e o cuidador mudarem de cidade, do interior para a capital do Estado, para ter acesso ao tratamento.

"No início tiveram dificuldade para entender que a água fazia mal, mas hoje eles dão suporte”. (C5)

"A família entende, eles não foram muito afetados...acho que a queixa maior deles é a distância, a saudade”. (C6)

"Sim, mas facilitou bastante pra pegar o transporte (a mudança de cidade), mas tive que romper um relacionamento amoroso". (C7)

A família que vivencia o cuidado à criança com DRC passa por um processo de reorganização da rotina ${ }^{1}$. Os pacientes com DRC, em programa regular de hemodiálise, enfrentam um regime terapêutico complexo e muitos têm dificuldade em gerir as restrições de líquidos e da dieta. Esta situação se torna ainda mais complexa quando se trata de crianças. As medidas de autocuidado, centram-se em duas dimensões: a redução do consumo de sódio e o controle da ingestão hídrica (p. 76) ${ }^{11}$. De acordo com as entrevistas, o suporte familiar é indispensável para a criança compreender a necessidade de tais cuidados para sua vida.

"Sim, houve mudança, principalmente na ingestão de sal e água”. (C8)

"Mudou muito devido as restrições que essa doença traz."(C9)

"Sim. Toda família aderiu a dieta. Às vezes até eu tomo pouca água”. (C3)

É possível identificar que as cuidadoras perceberam as alterações alimentares no seu dia a dia e que muitas famílias aderiram a dieta prescrita à criança como forma de facilitar a adesão a essas modificações, deixando assim seus hábitos alimentares. Porém, é importante ressaltar que algumas medidas se tornam inapropriadas e prejudiciais para a família, como podemos perceber nos relatos acima, especificamente na fala da cuidadora 3 , que mesmo sem necessidade reduziu a ingestão hídrica.

Considerando o tratamento integral a uma doença crônica ressalta-se que além das internações periódicas 
Santos HSS, et al. Repercussões de doença renal crônica na rotina familiar. Rev Ter Ocup Univ São Paulo. 2020 jan.-dez.;31(1-3):24-30.

e acompanhamento contínuo, o seu enfrentamento exige da família mais do que disponibilidade de tempo, requer também dedicação, reorientação das finanças, organização de tarefas na tentativa de reorganizar a vida a partir dessa nova circunstância ${ }^{3}$.

\section{Os dias mais preocupantes}

A doença crônica na infância e o tratamento contínuo acarretam forte tensão ao paciente e aos familiares, especialmente para os que assumem o papel de cuidador (p. 94 $)^{12}$, e diariamente é necessário manter uma rotina de cuidados, que no caso do tratamento de hemodiálise,

"Acordamos às 5 horas da manhã e o dia é todo voltado para a hemodiálise e descanso, pois todos ficamos bem cansados". (C2)

"A gente praticamente não dorme, temos que sair de lá 23:30 para chegar aqui no horário”. (C7)

Os relatos das cuidadoras demonstraram que há grande preocupação nos dias em que ocorre a hemodiálise, pois deixam em casa não apenas outras tarefas para serem realizadas, mas também a oportunidade de ofertarem cuidado aos outros filhos, cônjuge e demais familiares.

"Saio mais cedo de casa para poder chegar aqui no horário e tem que ter uma organização na casa, pois tem meu outro filho, meu esposo." (C1)

"Acordamos 03:00 horas da manhã e meu esposo vai trabalhar e nossas duas filhas ficam com parentes. Não sei quase nada do que acontece no dia lá, pois só retorno no fim da tarde." (C8)

Durante o tratamento de hemodiálise podem ocorrer complicações como hipotensão, cefaleias, náuseas e vômitos, tonturas e fadiga (p. 235) ${ }^{13}$. Assim sendo, a preocupação das cuidadoras é exacerbada em cada sessão de hemodiálise.

"Eu fico tensa. Sei como vai começar, mas não sei como vai terminar, já vi crianças morrerem e outras irem pra UTI". (C3)

"Me sinto preocupada, mas entrego tudo nas mãos de Deus". (C8)

\section{Os dias mais tranquilos}

Durante a semana, nos dias em que a hemodiálise não ocorre a rotina da família também sofre alterações.
"É um dia muito corrido, pois tenho que resolver outros problemas e ele fica muito cansado”. (C7)

"Há muitas atividades domésticas e do trabalho acumuladas para fazer”. (C9)

Foi identificado que para as cuidadoras e suas crianças que precisam deslocar-se do seu município de moradia até o local de tratamento, esses dias da semana são sinônimos de descanso.

“Acordamos mais tarde. Eu levo ele na escola...tenho mais tempo para mim. Descanso também”. (C5)

"É normal. Temos nossas atividades para fazer, temos que deixar tudo organizado também...limpar a casa, momento da escola, de comer e brincar". (C8)

As cuidadoras evidenciaram a preocupação de realizar atividades que envolvam as crianças como forma de proporcionar dias mais agradáveis apesar da rotina de tratamento.

"É tranquilo, vamos à igreja e ele vai para o colégio. Temos mais tempo também para brincar com ele, ir à casa da mamãe”. (C10)

"É o dia de fazer as coisas que não costumamos fazer, ele vai ao colégio, temos mais tempo para dormir e para ficar em família”. (C1)

\section{DISCUSSÃO}

A necessidade que a criança com DRC tem de realizar a hemodiálise traz alterações e na vida familiar, devido às necessidades intrínsecas desencadeadas pelo tratamento. Tais mudanças estão relacionadas ao processo de desestruturação e de modificação dos papéis desempenhados por cada membro, resultando em momentos conflituosos e na interferência do cotidiano que vão desde a desorganização familiar, da vida pessoal às manifestações de exaustão além de ser marcado por emoções negativas, pois é um processo acompanhado de sentimentos de descoberta, incertezas, receios e revolta ${ }^{14}$.

Os resultados apontaram que a maioria das entrevistadas foram as mães das crianças e se perceberam com a atribuição de desempenhar o papel de familiar cuidador. Ao surgir o conceito do familiar cuidador, é importante conhecer e explorar as percepções deste. Familiares 
cuidadores são aqueles que prestam cuidados a outro familiar de uma forma regular e não remunerada ${ }^{15,16}$.

Considera-se a mãe um membro significativo para representar a família, por participar ativamente da construção e da organização do cotidiano no domicílio. Em um estudo descritivo que analisou as dificuldades vivenciadas pela família e pela criança/adolescente com DRC, os resultados revelaram que do total de cuidadores participantes da pesquisa $80 \%(24)$ destes eram as mães ${ }^{17}$. Esse dado também foi encontrado em nossa pesquisa, onde $90 \%$ (9) das cuidadoras eram as próprias mães das crianças.

Quando a criança é acometida por uma doença grave, os pais experimentam transformações que significam a perda de algumas ocupações habitualmente desempenhadas por eles, passando a vivenciar uma nova realidade em que o foco passa a ser o tratamento do filho ${ }^{10}$. Isso significa se abster de ocupações como atividades instrumentais de vida diária (AIVD), lazer e educação, logo estas podem ser parcial ou integralmente prejudicadas.

Os relatos das cuidadoras explicitam que uma das primeiras mudanças ocorridas em suas rotinas foi o distanciamento da ocupação trabalho. Em uma pesquisa realizada em 2012 com familiares de crianças com doença crônica, foi constatado que uma mudança comum no ambiente familiar é o abandono do trabalho de um dos pais para acompanhar o filho durante a hospitalização, as consultas frequentes e nas realizações de exames ${ }^{10}$. Um estudo realizado com cuidadores observou que estes associaram as responsabilidades alusivas ao cuidado com anulações dos seus planos de vida e de trabalho ${ }^{18}$.

Os resultados apontaram preocupação das cuidadoras devido à ausência e fragilidade do relacionamento com os demais membros da família, em decorrência do tempo dedicado ao tratamento da criança com DRC. Em especial, relataram maior preocupação com a dificuldade de prover cuidados aos outros filhos, que nem sempre conseguem compreender a necessidade da ausência da mãe.

Pesquisa realizada em um hospital escola do Estado da Paraíba, evidenciaram que o distanciamento dos outros filhos é uma alteração frequente, e declarada pelas famílias entrevistadas como uma grande dificuldade e motivo de preocupação ${ }^{3}$.

A partir da análise das entrevistas observou-se que as alterações da dinâmica familiar se tornam mais impactantes nos dias em que ocorre o tratamento de hemodiálise. Parte disso deve-se à necessidade de deslocamento ao hospital, devido ao fato de que a maioria das crianças são advindas de municípios do interior do estado, algumas têm que lidar com horas de viagem para realizarem a hemodiálise, tornando o tratamento mais estressante e desgastante para a criança e a cuidadora.
Algumas famílias tiveram que mudar de cidade, mas apesar da mudança apresentar benefícios por facilitar o acesso ao tratamento, influenciou diretamente na quebra da rotina familiar e no relacionamento com os demais membros familiares.

Apesar das várias situações que favorecem o estresse, desânimo ou até mesmo a falta de esperança, observou-se nos relatos das cuidadoras a busca pelo conforto espiritual, muitas vezes utilizado como apoio emocional. Em estudo com ênfase nas estratégias utilizadas pelas famílias para lidar com a DRC e o tratamento, os dados sugerem que a religião e a espiritualidade são apontadas como vínculos apoiadores a fim de proporcionar conforto e alívio para as pessoas envolvidas no cuidado à pessoa com $\mathrm{DRC}^{19}$.

Os dias em que não ocorre o tratamento de hemodiálise são vivenciados e sentidos de forma particular pelas cuidadoras. Para os familiares que residem em outros municípios é sinônimo de um dia de descanso. Outras cuidadoras enfatizam que têm atividades pessoais para realizar ou mesmo antecipar a organização das tarefas domésticas para o dia seguinte, em que acompanharão seus filhos ao hospital. Alguns relatos referem à preocupação de proporcionar momentos de lazer, estudo, brincar entre os filhos, almoços em família a fim de amenizar os impactos negativos que os dias de tratamento configuram a rotina da família como um todo.

\section{CONCLUSÃO}

As repercussões da DRC na rotina familiar de crianças em tratamento de hemodiálise puderam ser analisadas a partir dessa pesquisa. Os relatos evidenciaram uma realidade difícil para as famílias de crianças em hemodiálise. Acreditase que essas famílias vivenciam não apenas a alteração em suas rotinas, mas a reorganização da estrutura familiar ocasionando perdas a todos os membros envolvidos.

Antes de enfrentar o procedimento de hemodiálise essas famílias enfrentam dificuldades relacionadas à falta de estrutura, deslocamento, falta de suporte familiar e financeiro. A alteração na rotina familiar é inevitável, tornando-se mais impactante nos dias em que ocorre o tratamento, mas a pesquisa também ressaltou que mesmo nos dias em que o tratamento de hemodiálise não ocorre, seus impactos e significados continuam presentes.

O longo tempo de espera para a realização do transplante torna esse processo mais sofrido e desgastante para a criança e sua família, e a possibilidade de transplante renal significa esperança de cura. Também significa que não será mais necessário o tratamento de hemodiálise e todas as implicações a ela relacionadas. Assim, tanto a criança como 
Santos HSS, et al. Repercussões de doença renal crônica na rotina familiar. Rev Ter Ocup Univ São Paulo. 2020 jan.-dez.;31(1-3):24-30.

seus familiares poderão desempenhar suas ocupações sem restrições e a rotina familiar passará a ser mais satisfatória.

Portanto, o conhecimento da realidade vivenciada pela família é importante para o desenvolvimento de estruturas mais sólidas para seu fortalecimento, em busca de superar os percalços existentes na vida das pessoas envolvidas no processo do adoecer da criança com doença renal crônica ${ }^{5}$.

Quanto as limitações no processo da pesquisa, podese citar o baixo número de participantes. Em novo estudo, seria pertinente um maior período para vinculação com os participantes e também visitas domiciliares a fim de observar melhor as rotinas familiares. Dessa forma, ressalta-se a importância em considerar a família como um cliente a ser cuidado, assegurando-lhe todo o acompanhamento necessário para ajudá-la a lidar com as dificuldades do adoecer ${ }^{20}$.
Quadro 1 - Caracterização dos cuidadores das crianças

\begin{tabular}{lcc}
\hline Cuidador principal & Idade & Parentesco com a criança \\
\hline Cuidadora 1 & 45 & Mãe \\
Cuidadora 2 & 35 & Mãe \\
Cuidadora 3 & 28 & Mãe \\
Cuidadora 4 & 28 & Mãe \\
Cuidadora 5 & 30 & Mãe \\
Cuidadora 6 & 32 & Mãe \\
Cuidadora 7 & 45 & Mãe \\
Cuidadora 8 & 41 & Mãe \\
Cuidadora 9 & 30 & Mãe \\
Cuidadora 10 & 56 & Avó paterna \\
\hline
\end{tabular}

Fonte: Autores da pesquisa

Participação dos autores: Heloisa Sâmella Santos dos Santos e Larice Pinto de Almeida- coleta, organização e análise dos dados, concepção e redação do texto; Fernanda do Socorro Carvalho da Silva- coleta de dados e revisão do texto; Elson Ferreira Costa- revisão do texto; Luísa Sousa Monteiro Oliveira- orientadora e responsável pela concepção da pesquisa e revisão do texto.

\section{REFERÊNCIAS}

1. Barbosa DC, Sousa FGM, Silva ACO, Silva IR, Silva DCM, Silva TP. Funcionalidade de famílias de mães cuidadoras de filhos com condição crônica. Cienc Cuid Saude 2011;10(4):731-38. doi: http://dx.doi.org/10.4025/cienccuidsaude.v10i4.18317

2. Pinho SR, Souza BSF, Cunha CCC, Costa DM, Nunes LN, Fonseca LCG, Lima MF, Mello RP, Hora TWJP, Silva VVC. Alterações comportamentais em crianças portadoras de enfermidades crônicas e suas repercussões na família: Hospital da Criança - Obras Sociais de Irmã Dulce, Salvador, BA. Rev Cienc Méd Biol. 2007;6(1):0716. doi: http://dx.doi.org/10.9771/cmbio.v6i1.4138

3. Silva MAS, Collet N, Silva KL, Moura FM. Cotidiano da família no enfrentamento da condição crônica na infância. Acta Paul Enferm. 2010; 23(3):359-65. doi: http://dx.doi.org/10.1590/ S0103-21002010000300008

4. Cavalcanti A, Silva Dutra FCM, Elui VMC (trad).American Occupational Therapy Association, A. Estrutura da prática da Terapia Ocupacional: domínio e processo (3ed.). Rev Ter Ocup Univ São Paulo. 2015; 26(ed.esp.):1-49. doi: http://dx.doi.org/ 10.11606/issn.2238-6149.v26iespp1-49

5. Turato ER. Métodos qualitativos e quantitativos na área da saúde: definições, diferenças e seus objetos de pesquisa. Rev Saúde Pública. 2005;39(3):507-14. doi: http://dx.doi. org/10.1590/S0034-89102005000300025

6. Fundação Santa Casa de Misericórdia do Pará. Santa Casa do Pará - Assistência e ensino. Pará; 2018 [citado 31 jul. 2018]. Disponível em: www.santacasa.pa.gov.br.
7. Bardin L. Análise de conteúdo. Portugal: Lisboa: Edições 70; 2009.

8. Witt WP, De Leire T. A Family Perspective on Population Health: The case of child health and the family. WMJ.2009; 108(5):240-45 [citado 5 maio 2019]. Disponível em: https:// www.ncbi.nlm.nih.gov/pmc/articles/PMC2871389/

9. Chiattone HBC. A criança e a morte. In: Angerami-Camom VA, organizador. E a psicologia entrou no hospital. São Paulo: Pioneira Thomson Learning; 2001. p. 69-102.

10. Nóbrega VM, Reicherti APS, Silva KL, Coutinho SED, Collet N. Imposições e conflitos no cotidiano das famílias de crianças com doenças crônicas. Esc Anna Nery. 2012;16(4):781-8. doi: http://dx.doi.org/10.1590/81452010200400020S1414.

11. Vieira MA, Dupas G, Ferreira NMLA. Doença renal crônica: conhecendo a experiência da criança. Esc Anna Nery. 2009;13(1):74-83. doi:http://dx.doi.org/10.1590/5141481452009000100011 .

12. Meira B, Vale T. Nível de estresse das acompanhantes de pacientes internados no setor de pediátrico e os aspectos sócio emocionais inter-relacionados. In: Neme C, Rodrigues O, organizador. Psicologia da saúde: perspectivas interdisciplinares. São Carlos: Rima; 2003. p. 193-245.

13. Fermi MRV. Diálise para Enfermagem: Guia prático. 2a ed. Rio Janeiro: Guanabara Koogan; 2010.

14. Almeida A, Pacheco E, Mota L. A família: o primeiro suporte ao insuficiente renal crônico. Rev Nephros. 2010;11:19-30. 
15. Caetano JPM, Fernandes MV, Marcon SS, Decesaro, MN. Refletindo sobre as relações famílias e os sentimentos aflorados no enfrentamento da doença crônica. Ciênc Cuid Saúde. 2011;10(4):845-52. doi: 104025/cienccuidsaude.v20i4.18331.

16. Wegner W, Pedro ENR. Os múltiplos papéis sociais de mulheres cuidadoras-leigas de crianças hospitalizadas. Rev Gaúcha Enferm. 2010; 31(2):335-42. doi: http://dx.doi.org/10.1590/ S1983-14472010000200019.

17. Abrahão SS, Ricas J, Andrade DF, Pompeu FC, Chamahum L, Araújo TM, et al. Dificuldades vivenciadas pela família e pela criança/adolescente com doença renal crônica. J Bras Nefrol. 2010;32(1):18-22. doi: http://dx.doi.org/10.1590/ S0101-28002010000100004
18. Jorge CF, Toldrá RC. Percepção dos cuidadores sobre a experiência de cuidar dos familiares e a relação com a equipe profissional no contexto da hospitalização. Rev Ter Ocup Univ São Paulo. 2017; 28(3):271-80. doi: 10.11606/issn.2238-6149. v28i3p271-280

19. Burille A, Zillmer JGV, Swarowsky GE, Schwartz E, Muniz RM, Santos BP, et al. Os vínculos apoiadores como estratégia das famílias para lidar com a doença renal crônica e o tratamento. Rev Enferm UFPE. 2010; 4(1):106-11. doi: 10.5205/1981-8963-v4i1a5839p106-111-2010

20. Salgado CL, Branco MMMC, Machado PMA. A Família no processo de cuidar do paciente com doença renal crônica. São Luís: Edufma; 2016. Disponível em: https://ares.unasus. gov.br/acervo/handle/ARES/7479 